\title{
Heterogeneity of Sonic Hedgehog Response Dynamics and Fate Specification in Single Neural Progenitors
}

\author{
Fengzhu Xiong ${ }^{1,3}$, Andrea R. Tentner ${ }^{1,3}$, Tom W. Hiscock ${ }^{1}$, Peng Huang ${ }^{2}$, Sean G. Megason ${ }^{1, \star}$ \\ ${ }^{1}$ Department of Systems Biology, Harvard Medical School, Boston, MA, USA \\ ${ }^{2}$ Department of Biochemistry \& Molecular Biology, University of Calgary, Calgary, Alberta, Canada \\ ${ }^{3}$ Co-first authors \\ "correspondence: megason@hms.harvard.edu
}

\section{SUMMARY}

During neural tube patterning, a gradient of Sonic hedgehog (Shh) signaling specifies ventral progenitor fates. The cellular response to Shh is processed through a genetic regulatory network (GRN) to code distinct fate decisions. This process integrates Shh response level, duration and other inputs and is affected by noise in signaling and cell position. How reliably a single cell's Shh response profile predicts its fate choice is unclear. Here we use live imaging to track neural progenitors that carry both Shh and fate reporters in zebrafish embryos. We found that there is significant heterogeneity between Shh response and fate choice in single cells. We quantitatively modeled reporter intensities to obtain single cell response levels over time and systematically determined their correlation with multiple models of cell fate specification. Our input-output analysis shows that while no single metric perfectly predicts fate choices, the maximal Shh response level correlates best overall with progenitor fate choices across the anterior-posterior axis.

\section{KEYWORDS}

Shh, Neural tube, response, fate, heterogeneity, single cell, GRN

\section{INTRODUCTION}

Morphogens pattern tissue by acting over space in a concentration dependent manner (Wolpert 1969). Biochemically, morphogen molecules may either regulate transcription directly (Driever and Nusslein-Volhard, 1989), or through a specific transducer following a cascade of intracellular interactions - a signaling pathway. The latter involves additional, potentially non- 
linear steps of signaling. A prominent example of morphogen patterning is the vertebrate ventral neural tube where the morphogen Sonic hedgehog (Shh), produced from the notochord and floor plate, forms an extracellular gradient. Through its transcriptional effector Gli, this Shh gradient specifies different neural progenitor domains along the ventral-dorsal axis (Jessell 2000, Figure 1A). In this system, Gli activity profiles (cellular responses) regulate the expression of multiple homeobox transcription factors (fate genes) (Briscoe et al., 2000). These fate genes regulate their own and each other's expression as a genetic regulatory network (GRN); this network finally settles into one of several discrete and stable states that correspond to different neuronal fates (Lek et al., 2010; Balaskas et al., 2012). On the basis of population-level observations of the Shh pathway and transcriptional network, different aspects of the Shh signaling/Gli activity profile have been proposed to control cell fate choices: e.g., threshold levels (presumably by different affinities between Gli and fate gene enhancers, Ericson et al., 1997; Oosterveen et al., 2012), duration of response (sustained Gli levels under the adaptive negative feedback of the Patched [Ptc] receptor, Dessaud et al., 2007) and the time-integrated response level (Dessaud et al., 2010). As these response dynamics are integrated through the GRN towards fate decisions, inherent noise in gene expression (Rosenfeld et al., 2005) may generate heterogeneity in the response-fate correlation, despite the ability of the GRN to increase robustness (Balaskas et al., 2012). In addition, our recent work reveals extensive cell movement (positional noise) and a role of cell sorting in refining the boundaries of fate domains in the zebrafish neural tube after cells are specified (Xiong et al., 2013), raising the questions 1) if the interpretation of Shh signal is noisy; and 2) whether precise cell fate choice is necessary since patterning can be corrected after specification at least in zebrafish. While populational level studies using genetic perturbations have been important in dissecting the GRN structure of Shh interpretation, they do not address these questions as the naturally occurring heterogeneity is usually not separable from noise introduced by perturbation and thus is averaged out altogether in analysis. Single cell observations using live imaging with reporters, on the other hand, can be very effective in measuring endogenous variation by simultaneously recording how neural progenitors respond to Shh and make fate choices in vivo (Xiong and Megason, 2015).

\section{RESULTS}

Live tracking of individual neural progenitor fate choices in zebrafish embryos 
To enable a direct, single-cell quantification of Shh response dynamics and fate choice, we developed an imaging protocol using double transgenic reporters along with a nuclear or membrane localized EBFP2 for cell tracking (Figure 1B). We combine the tgBAC(ptch2:kaede) reporter whose Kaede expression reports Shh response (Huang et al., 2012) and a second transgenic reporter (e.g., tg(nkx2.2a:mgfp) [Ng et al, 2005], tg(olig2:gfp) [Shin et al, 2003], Figure 1C) whose expression levels indicate different fate choices (Xiong et al., 2013). Movies with good spatial-temporal coverage of the neural plate/tube (e.g., Movies S1) were analyzed by single cell tracking in GoFigure2, an open-source software we previously developed for image analysis (Xiong et al., 2013). By manually placing a seed at the center of the same cell at each time-point in the dataset, we generated a series of spherical segmentations inside the cell, which capture the fluorescence intensity of each reporter and the position of the tracked cell over time. Together, this time-dependent positional and reporter intensity information represents what we call a 'neural progenitor track' (Figures 1D-E, Movie S2). We analyzed over 200 randomly chosen tracked cells to cover the ventral progenitor types in the zebrafish neural tube: the medial floor plate cells (MFPs), lateral floor plate cells (LFPS), motor neuron progenitors (pMNs) and "More Dorsal" cells (e.g., p2,p1,p0 progenitors) (Figure 1A; Xiong et al., 2013). These cell fates form distinct domains highly conserved among vertebrates and prescribe the pattern of neurogenesis (Jessell 2000; Lewis and Eisen, 2003). In zebrafish, MFPs along with the notochord are the source cells of Shh; LFPs, pMNs and More Dorsal cells depend on Shh signaling for correct specification (Park et al., 2004; Huang et al., 2012). Taking advantage of the reporter expression and the stereotypic position at the end time point of each track, we identified and assigned different fates to the tracked neural progenitors (Xiong et al., 2013). We imaged cells from both the anterior spinal cord (approximately somite levels 4-9) as well as the posterior spinal cord (approximately somite levels 9-14). The shape, size and morphogenetic movements of both the Shh sending and receiving tissues vary along the AP axis (Xiong et al., 2013), thus allowing us to sample a diversity of input-output (i.e., Shh response-fate choice) relationships. 
bioRxiv preprint doi: https://doi.org/10.1101/412858; this version posted September 10,2018 . The copyright holder for this preprint (which was not certified by peer review) is the author/funder, who has granted bioRxiv a license to display the preprint in perpetuity. It is made available under aCC-BY-NC-ND 4.0 International license.
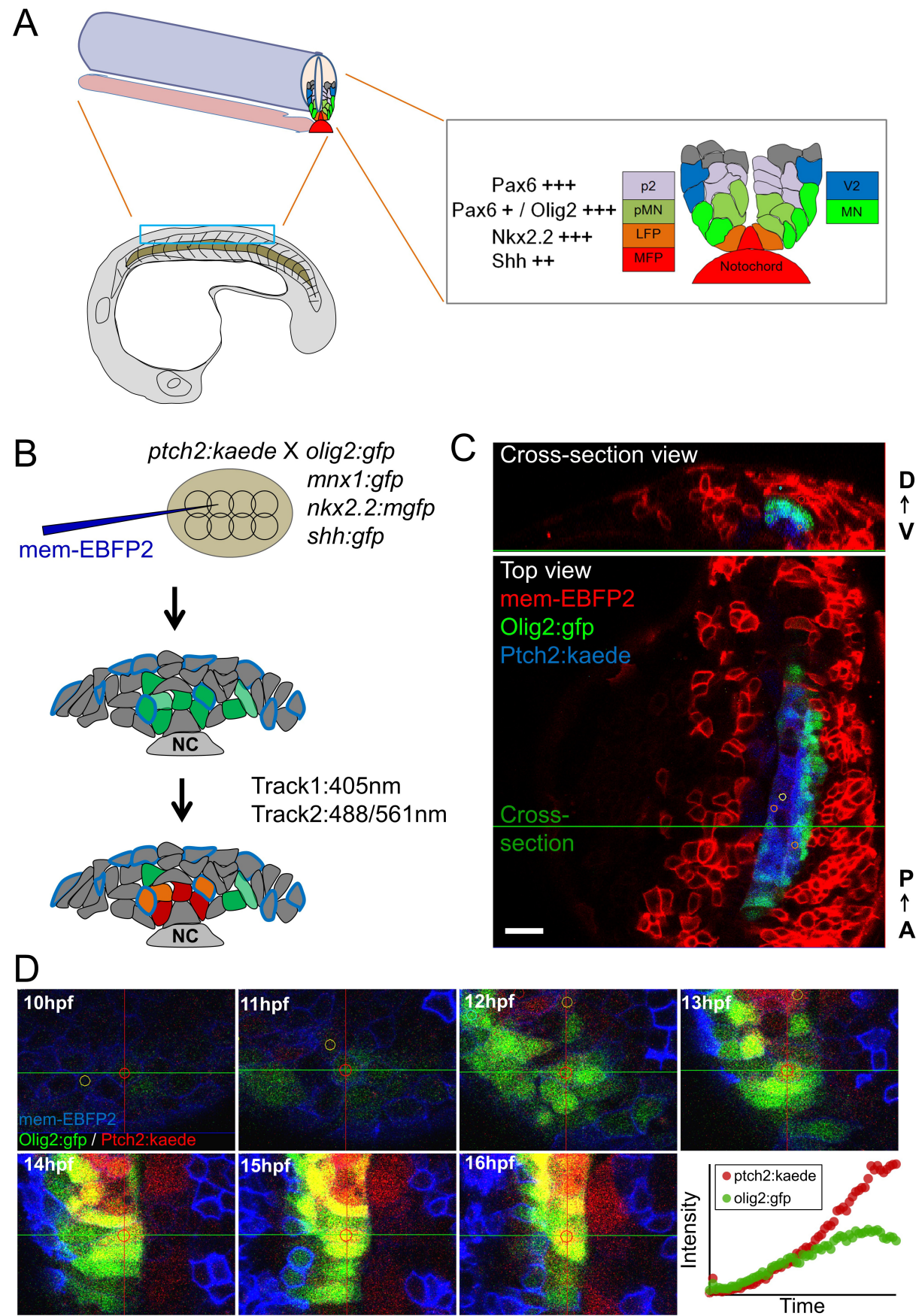

E
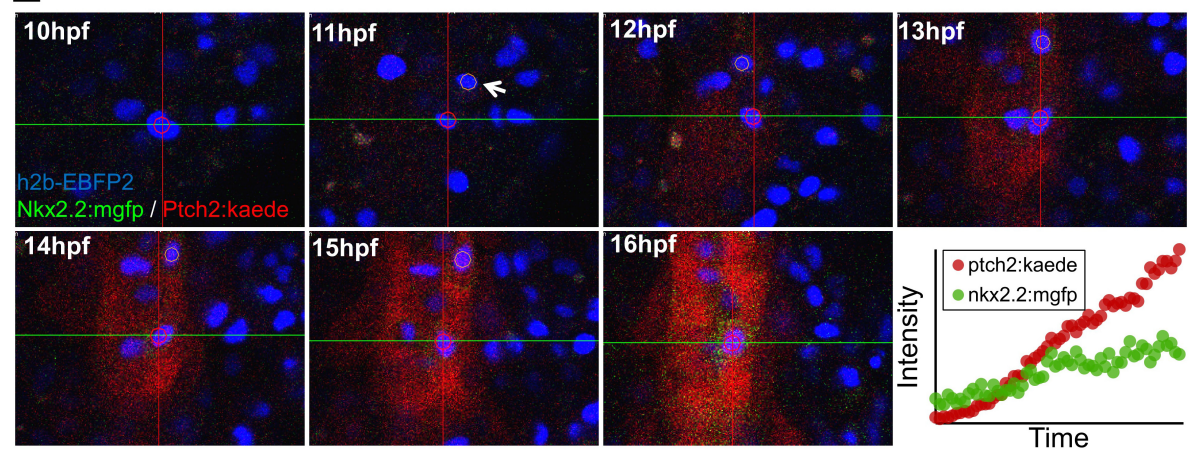
Figure 1. Imaging zebrafish neural tube development and single cell tracks of different fates.

(A) Schematic of ventral neural tube anatomy and pattern in zebrafish (partially adapted from Xiong et al., 2013). MFP, medial floor plate; LFP, lateral floor plate; pMN, motor neuron progenitors (MN, motor neurons); p2, V2 interneuron progenitors (V2, V2 interneurons).

(B) Labeling (at 8-16 cell stage) and imaging (at neural plate, neural keel and neural tube stages) scheme for acquiring three channels at the same time. The 405 laser in track 1 excites the BFP cell tracking marker as well as converting Kaede(Green) to Kaede(Red). NC, notochord.

(C) Example data. Small circles on the image show manual segmentations generated oncells using GoFigure2 software. D-V, dorsal-ventral; A-P, anterior-posterior. Scale bar: $20 \mu \mathrm{m}$.

(D) A pMN track in a mem-ebfp2, ptch2:kaedelolig2:gfp dataset. Circles in the cells show a 2D view of the spherical segmentations generated using GoFigure2 software.

(E) A LFP track in a h2b-ebfp2, ptch2:kaede/nkx2.2:mgfp dataset. Arrow indicates a sister cell of the centered track. Note that while this transgenic reporter has a membrane tag, the spherical segmentation is still capable of measuring the much weaker cytoplasmic fluorescent signal. See also Movie S1.

Heterogeneity is pervasive in Shh response dynamics and fate choice in single cells Our set of $\sim 200$ single cell tracks provides a first glimpse into the dynamics of Shh interpretation in vivo. The main new insights come from being able to observe the dynamics of two nodes of the GRN in the same live cell, namely a Shh response reporter (ptch2:kaede) and a fate reporter. To compare our data with previous studies, we first analyzed the average Shh response behavior of cells within each fate group (calculated by simple averaging of single cell tracks within each of the different fates). In both the anterior and posterior spinal cord in zebrafish (Movie S3 and S4, respectively), the average LFP shows the fastest increase of Kaede intensity, while the average pMN shows intermediate increase and the average More Dorsal cell is the slowest (Figures 2A,C). Accordingly, the olig2:gfp reporter is highly expressed in the average pMN but comes to a plateau after initial expression in the average LFP (Figures 2B,D). These data are consistent with observations that these cell types require different levels of Shh for specification (Ericson et al., 1997; Park et al., 2004) and that in amniotes the p3 progenitors (equivalent to LFPs in fish) initially express the pMN marker olig2 but then shift to express $n k x 2.2$ with continued and/or increasing Shh exposure (Jeong and McMahon, 2005; Dessaud et al., 2007).

We next ask whether single cells behave stereotypically like the averaged cell. Strikingly, at the single cell level, we observed high variability in Shh-signaling reporter dynamics across 
cells of the same fate and some overlap of cells that finally take on different fates (Figure 2A'-D'). There are examples of cells that have similar ptch2:kaede traces but distinct fate reporter traces (e.g., in Figure 2E, track 1 vs. 2, 3 vs. 4), as well as cells with very different ptch2:kaede traces but similar fate reporter traces (e.g., in Figure 2E, track 1 vs. 3). Across all datasets, we found $30 \%(17 / 56)$ of pMN cells have a ptch2:kaede trajectory that is similar to the average ptch2:kaede trajectory of corresponding LFP population ( $>50 \%$ of time points within $\pm 1 \times$ S.D. of the average LFP track). On the other hand, 38\% (11/29) of LFPs have a ptch2:kaede trajectory similar to pMNs. We also find a small overlap of ptch2:kaede trajectory between pMNs and More Dorsal cells (4/56 pMNs are similar to the average More Dorsal, 2/68 More Dorsal cells are similar to the average pMN). LFPs and More Dorsal cells are distinct (0/29 LFPs similar to More Dorsal, 0/68 More Dorsal similar to LFPs). These data show that single cells have a high degree of heterogeneity in the correlation between Shh response and fate choice. Cells that choose the same fate could have distinct Shh responses and cells that have very similar Shh responses may make different fate choices. 

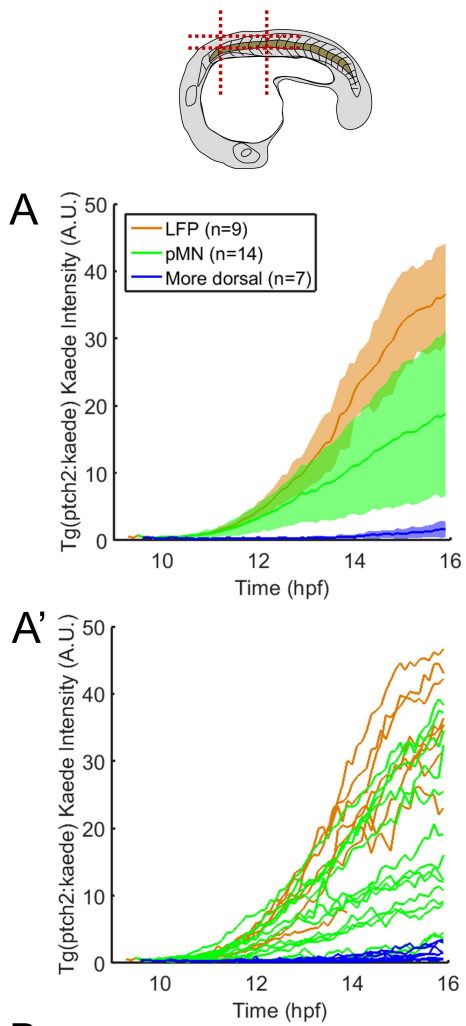

B

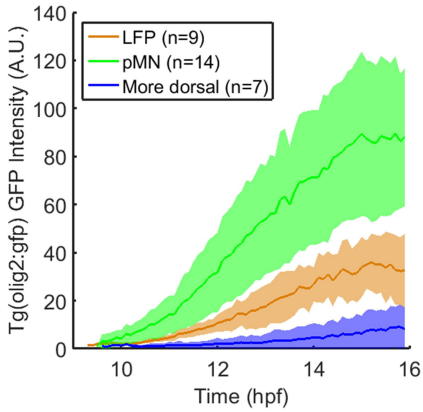

B'

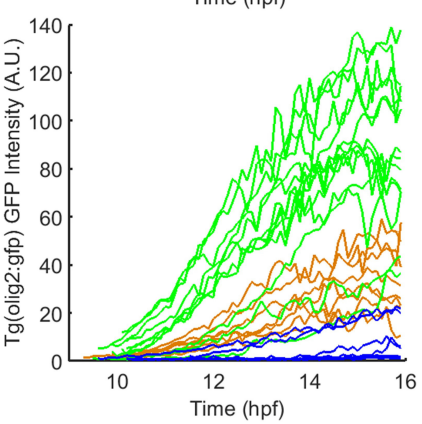

E

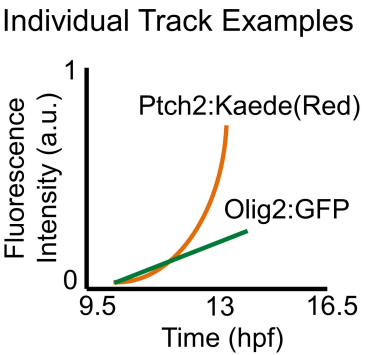

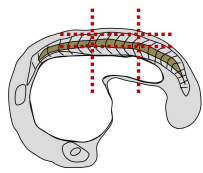

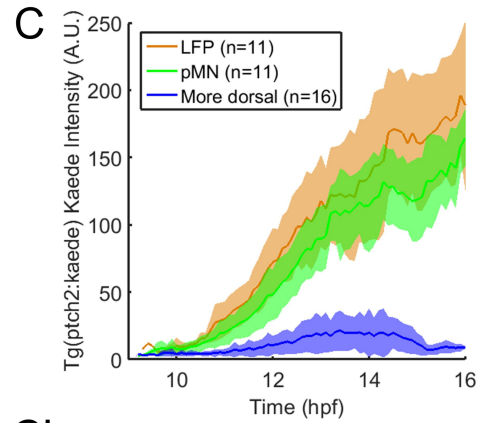

C'
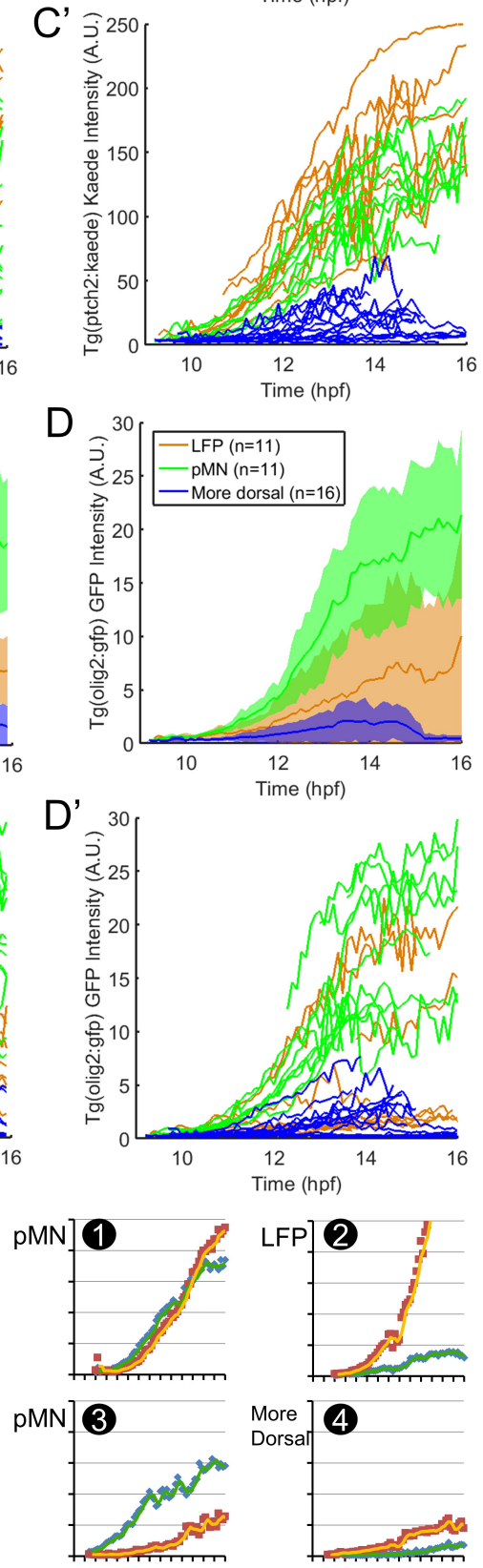
Figure 2. Dynamics and heterogeneity of Shh response and fate reporters in average cells and single cells. (A-D) Average and single (A'-D') tracks from a ptch2:kaede/olig2:gfp movie focusing on the anterior spinal cord (indicated by the schematic, A-B), and the posterior spinal cord (C-D), respectively. Colored shades around the average tracks represent \pm S.D. Individual ptch2:kaede traces $\left(A^{\prime}, C^{\prime}\right)$ show significant overlap between fates. Note that intensity units are not comparable between different datasets (e.g., between $A$ and $C$ ) due to variation in expression, sample depth, mounting position, and imaging settings. The average tracks appear noisier before $10 \mathrm{hpf}$ and after $15 \mathrm{hpf}$ due to some single cell tracks not being long enough temporally (e.g., a tracked cell leaves the field of view) therefore fewer data points are available for averaging at the ends. LFP, Lateral floor plate cells; pMN, motor neuron progenitors. See also Movies S3,4.

(E) Example single tracks that show very similar ptch2:kaede response dynamics but very different olig2:gfp expression, and vice versa. The schematic shows the axis ranges and labels, and legends for the two reporter traces.

\section{Quantifying reporter activity dynamics from fluorescent intensity tracks}

Given the apparent heterogeneity and overlap in the single cell track data, are there certain features in the reporter dynamics that predict fate outcome more accurately? In other words, are the apparently very similar ptch2:kaede tracks (as seen in Figure 2E) different in a certain way that could explain their distinct fate choices? Our tracks offer us an opportunity to address this question by quantifying Shh signaling activity, namely the transcription rate of the ptch2 reporter, from the fluorescence intensities. However, the intensity measurements must be handled carefully and interpreted with consideration of experimental limitations to provide useful insights.

First, while the fluorescence intensity levels measured in single cells by image segmentation are in principle proportional to the reporter protein concentration (Figure 3A), the acquisition and analysis processes may introduce several errors: bleaching, bleed-through, saturation, and segmentation measurement error. Our sampling frequency is lower here than our previous work where bleaching was not significant (Xiong et al., 2013). To account for bleedthrough, which refers to the phenomenon of signal from one fluorophore contributing to another during simultaneous acquisition, we applied a percentage subtraction from each channel after determining the bleed-through ratio using all data points (methods). To account for saturation, which refers to signal intensity exceeding the dynamic range of detection causing an artificial signal plateau, we removed data points where the fraction of saturated pixels exceeds $5 \%$ within segmented cells (methods). To handle noise that arises from random fluctuations of segmentation measurement between time points (technical errors), we applied a moving- 
average smoothing to the tracks over windows of 6 time points (36min, see Figure 6C for effects of varying this time window). This operation causes some short-time scale features of the tracks (which are otherwise not possible to analyze due to technical errors in the measurements) to be lost, but it preserves trends on longer time intervals (Figure 3B). We further confirmed the mitigation of the impact of technical errors by re-tracking the same cells, where the average variation of intensity measurement over 6 time points is consistently <5\% (data not shown). Together this pipeline transforms raw tracks to an intensity track that reasonably approximates the reporter protein concentration dynamics in single cells.

Second, in order to estimate the cellular Shh response/Gli activity dynamics (reflected by the transcription dynamics at the ptch2:kaede reporter), we investigated the relationship between fluorescent intensity $(\boldsymbol{I})$ and the transcription rate of kaede $(\boldsymbol{x})$ (Elowitz and Leibler, 2000). Since Kaede and GFP proteins can be considered as stable on the time-scale of our experiments (Ando et al., 2002; data not shown), assuming a constant coefficient ( $\left.\boldsymbol{c}_{1}\right)$ for the rate of Kaede translation and maturation as a function of kaede mRNA level $(\boldsymbol{m})$, the intensity change rate of Kaede (Figure $3 C$ ) is given by:

$$
\frac{d I}{d t}=c_{1} m
$$

$\boldsymbol{m}$ is in turn a function of transcription and mRNA stability, given in the form of half-life $\left(\boldsymbol{t}_{1 / 2}\right)$ :

$$
\frac{d m}{d t}=x-m \frac{\ln 2}{t_{1 / 2}}
$$

Combining (1) and (2) we have a simplified relationship between measured fluorescence intensity and Shh response (represented by $\boldsymbol{x}$ ):

$$
c x=\frac{d^{2} I}{d t^{2}}+\frac{d I}{d t} \cdot \frac{\ln 2}{t_{1 / 2}}
$$

$\boldsymbol{c}$ is a constant. Note that the fluorescent signal is time delayed due to lags caused by transcription, translation, and maturation of the fluorescent protein, therefore our raw tracks do not show reporter responses (transcription) in real time. Importantly, use of destabilized RNA or proteins would not reduce this lag, but only diminish reporter intensity thus making their measurement noisier. Furthermore, because of the time and detection sensitivity differences between Kaede and GFP, we are prevented from cross-correlating the two reporter channels on the same time axis. Despite these limitations, equation (3) still allows us to estimate the Shh response dynamics in each single cell and compare that between different cell fates within each 
dataset.
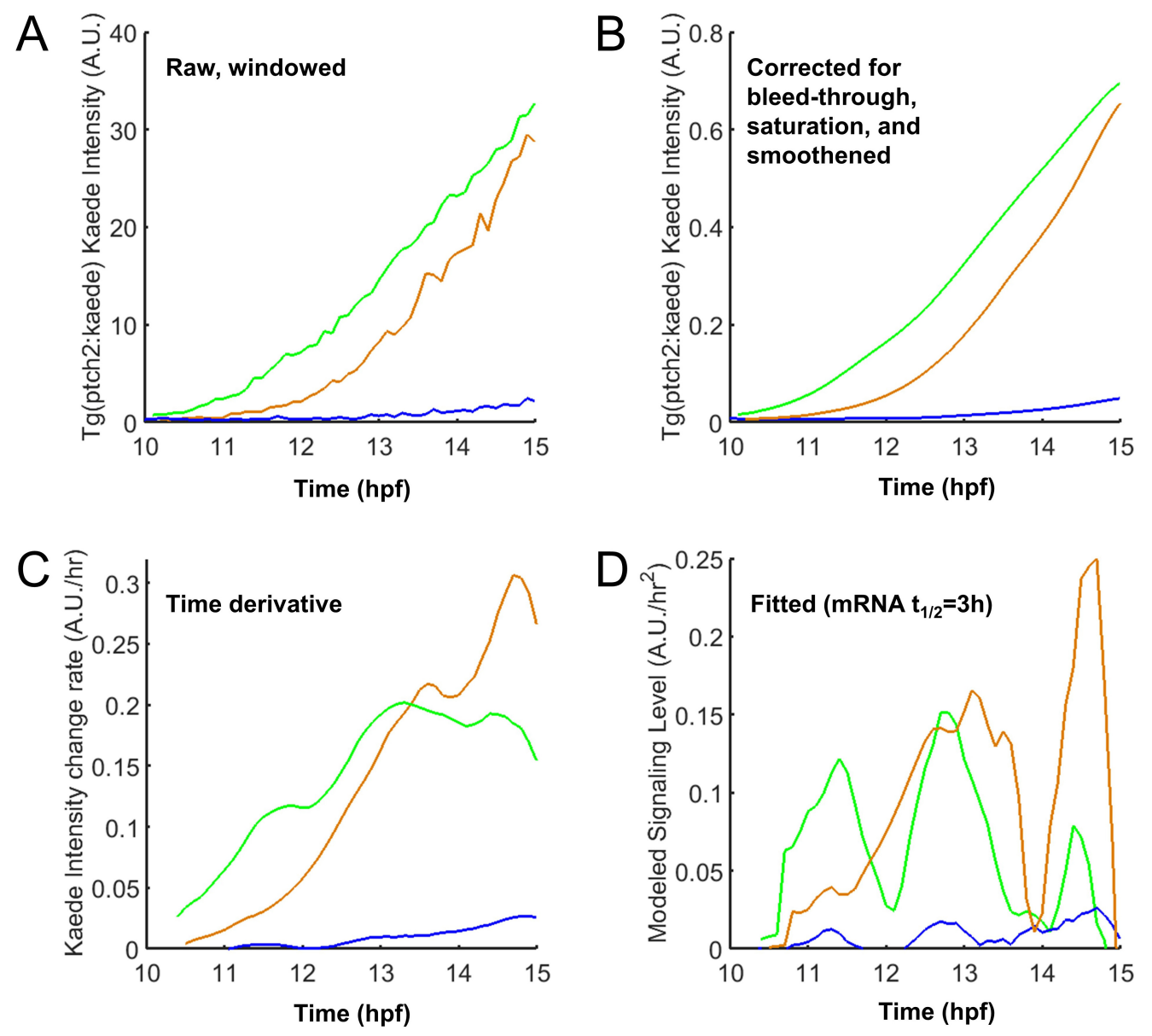

Figure 3. Estimation of dynamic reporter activity from fluorescence intensity measurements

(A) Example raw tracks (a pMN[green], a LFP[brown] and a More Dorsal cell[blue] each). The tracks were tailored (windowed) to contain only data points between 10 and $15 \mathrm{hpf}$ as this time window is best covered in the tracks. Before $10 \mathrm{hpf}$ the signal is low and by $15 \mathrm{hpf}$ most progenitors have become specified (Xiong et al., 2013).

(B) Tracks in (A) after multiple filter corrections as described in the text. Main trends over long time windows ( $>0.5$ hours) are preserved while short fluctuations are removed by a moving-average smoothing operation.

(C) Rate of change in intensity by the first time derivative of (B).

(D) Modeled signaling level (ptch2:kaede transcription rate) of the same tracks using equation (3). Note the different peak height and duration of different cells. Varying the half-life value between 1 to 5 hours does not substantially change these results (data not shown).

To validate equation (3) and estimate reporter mRNA half-life $\left(\boldsymbol{t}_{1 / 2}\right)$ in vivo, we first applied a heat-shock pulse on transgenic embryos with a hsp70l:EGFP transgene (Figures 4A; Halloran et al., 2000, constructed with an SV40 polyadenylation sequence like the ptch2:kaede 
transgene). This causes a defined pulse of transcriptional output at the reporter (Rieger et al., 2005). According to equation (3), the GFP intensity change that follows should reflect the exponential decay of the gfp mRNA. Indeed, single cell tracks we generated provide an excellent fit with the model's prediction (Figure 4B), allowing us to estimate the mRNA half-life to be around 1.7 hours. Next, we performed in situ hybridization of kaede after sequential chemical inhibition of Shh response using cyclopamine (thereby stopping new kaede mRNA synthesis) and compared the overall mRNA signal with controls for signal level over a series of time points (Figure 4C; Huang et al., 2012). This method suggests a 3-hour mRNA half-life. The two estimations are thus congruent on an in vivo half-life of fluorescent reporter mRNAs somewhere between 1-4 hours (effects of varying this parameter are assessed in Figure 6B). These data support the validity of equation (3) and enable us to use it and the estimated half-life to infer the dynamics of the ptch2 transcription rate (i.e. Shh signaling response) in our single neural progenitor tracks (Figure 3D). Note that as equation (3) contains secondary time derivatives of the intensity, this fitting is prone to measurement noise and requires good time resolution. However, due to our smoothing operation, sharp and short-time response changes have been filtered out. Our subsequent comparisons only consider those features that span over at least 0.5-hour windows (e.g., the peaks and valleys seen in Figure 3D) which are comprised of at least 5 time-point measurements and describe trends that can readily be seen in the raw intensity track (Figure 3B). This compromise minimizes the impact of technical errors in the analysis at the expense of model resolution. 

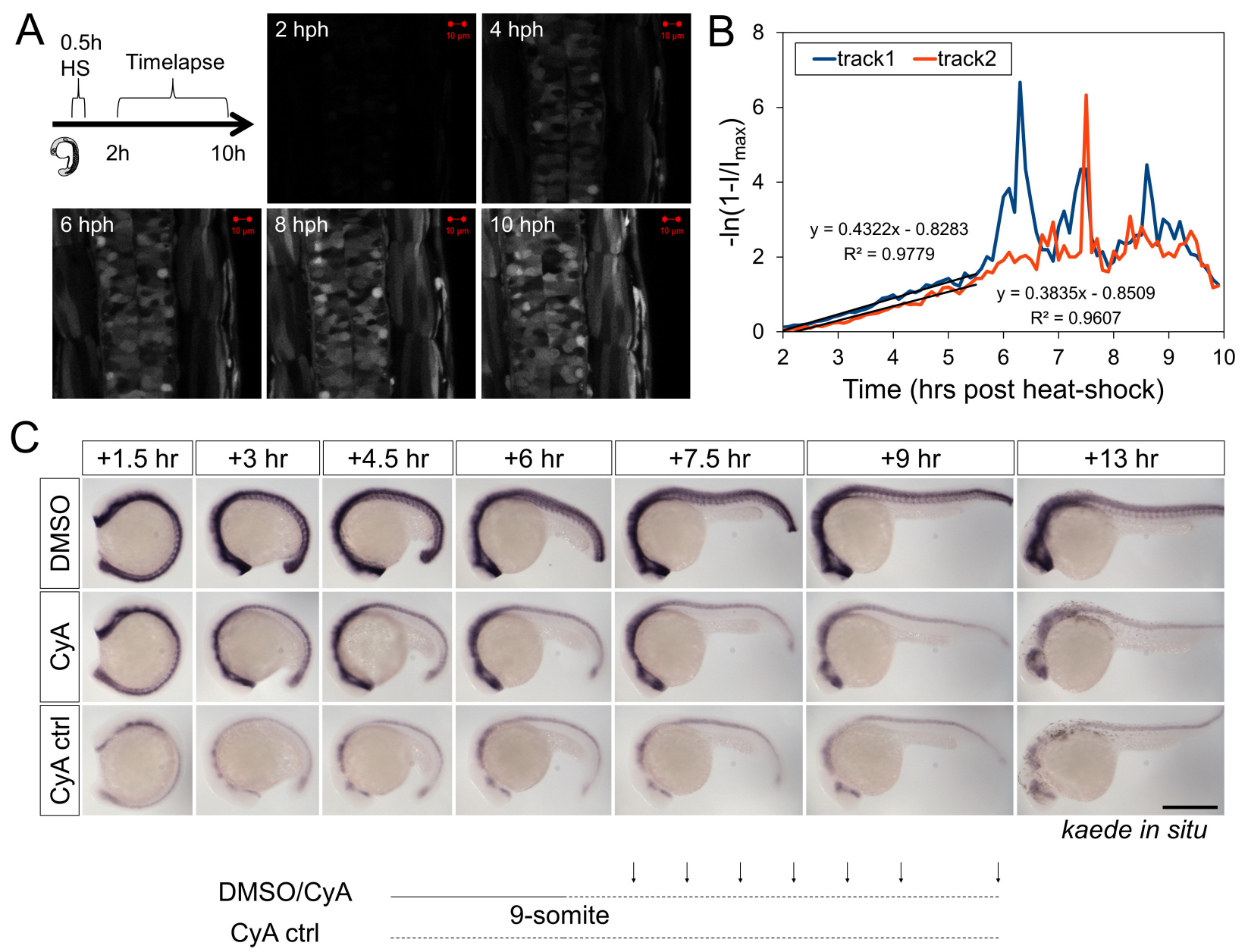

Figure 4. Estimation of fluorescent reporter mRNA half-life.

(A) Heat-shock pulse induction of GFP and timelapse imaging (schematic). 24hpf $t g$ (hsp70l:EGFP) embryos were shocked for 30 minutes in pre-warmed $37^{\circ} \mathrm{C}$ egg water in a heat block. Imaging began 2 hrs post heat-shock (hph, when the fluorescence was first detectable).

(B) Cell tracks of hsp70l:EGFP to validate equation (3) and estimate mRNA stability. Assuming that the heat-shock between $t=0$ and $t=0.5$ causes a transient pulse of mRNA production, the intensity track then covers the simple decay period of mRNA (as no more mRNA is produced after the pulse). The decay is exponential according to equation (3) and governed by the mRNA half-life. The fluorescent intensity increase rate then should correspondingly decrease exponentially towards a plateau value, predicting a linear relationship between time and $\operatorname{In}\left(1-I / I_{\max }\right)$, in which $\boldsymbol{I}$ is raw intensity and $I_{\max }$ is the plateau value of $\boldsymbol{I}$ in later times (when no more mRNA is left and new fluorescent protein production stops). This predicted linear relationship is found for the tracks suggesting the simple model correctly describes the process. The slope of the lines $(\sim 0.4)$ suggests the mRNA half-life of hsp70l:egfp to be $\sim 1.7$ hours. As $I$ approaches $I_{\max }$, the quantity of $I n\left(1-I / I_{\max }\right)$ becomes sensitive to small fluctuations in $I$, resulting in the noisy spikes seen after 6 hours. This portion of the data was not used in the fitting. (C) Estimation of Kaede mRNA stability using in situ hybridization. CyA, cyclopamine, an antagonist of Shh 
signaling that represses ptch2 promoter output. When soaked in CyA (CyA ctrl), the embryos exhibit a low basal level of Kaede expression in the neural tube. When CyA was added around 9-somite stage and embryos followed over time, the high level of Kaede mRNA as seen in the DMSO control was not maintained but decayed towards the basal level. In the trunk/tail neural tube (but not in the head), the level became similar to basal at +7.5 hours by comparing the CyA and CyA ctrl images. This result suggests Kaede mRNA is around for more than 6 hours after inhibition of signaling, with a half-life of about 3 hours. Scale bar: $400 \mu \mathrm{m}$.

\section{Correlation of different aspects of Shh response with fate outcome}

To compare the dynamic features of morphogen response in terms of their correlation to cell fate outcome, we started with metrics that have been reported to specify LFP, pMN, and More Dorsal cells in a graded manner. Using cell tracks from datasets as in Figure 2 for quantification, and performing analysis as in Figure 3, we calculated maximum transient response level (the peak Shh response within the track), average response level (average Shh response over 10-15hpf), and response time of Shh response (the amount of time with an above basal Shh response) in the tracked time windows. To give a visual indication of how well each metric correlates with the graded fate outcome, we ranked single cell tracks by the value of each metric high-to-low to make a vertical "French flag": the sharper the flag, the stronger the correlation. Along with the flag we calculate the mean percentage of correct fate prediction at a threshold value for each metric (methods). A value near 50\% (coin toss) would suggest the metric does not correlate with fate choice at all, whereas $100 \%$ would suggest the metric makes perfect prediction. For example, when we use two late-stage cell position metrics, the lateral-medial/dorsal-ventral (LMDV, this distance refers to the axis along which cells are being patterned which is initially lateral-medial because patterning begins at the neural plate which then gradually transitions to dorsal-ventral as the neural tube is formed) should do well in the flag correlation while the anterior-posterior (AP) position should do poorly since we are trying to predict fates across the DV axis (Figures 5A). Indeed, we find that the LMDV position is highly correlated with cell fates. For the anterior tracks, the LMDV distance is consistently $>90 \%$ correlated while for the posterior tracks the LMDV distance is more poorly correlated at earlier stages but becomes better at later times consistent with a necessary role for cell sorting in sharpening fate domain boundaries over time (Xiong et al., 2013). In contrast and as expected, the AP distances never correlate with fate choices in the anterior or posterior spinal cord (all around 50\%). The diversity of tracks and tissue geometry both within and across the anterior and posterior regions of the 
spinal cord (Figure 5B) enables us to conduct a rigorous comparison of how Shh response dynamics correlate with fate choice.

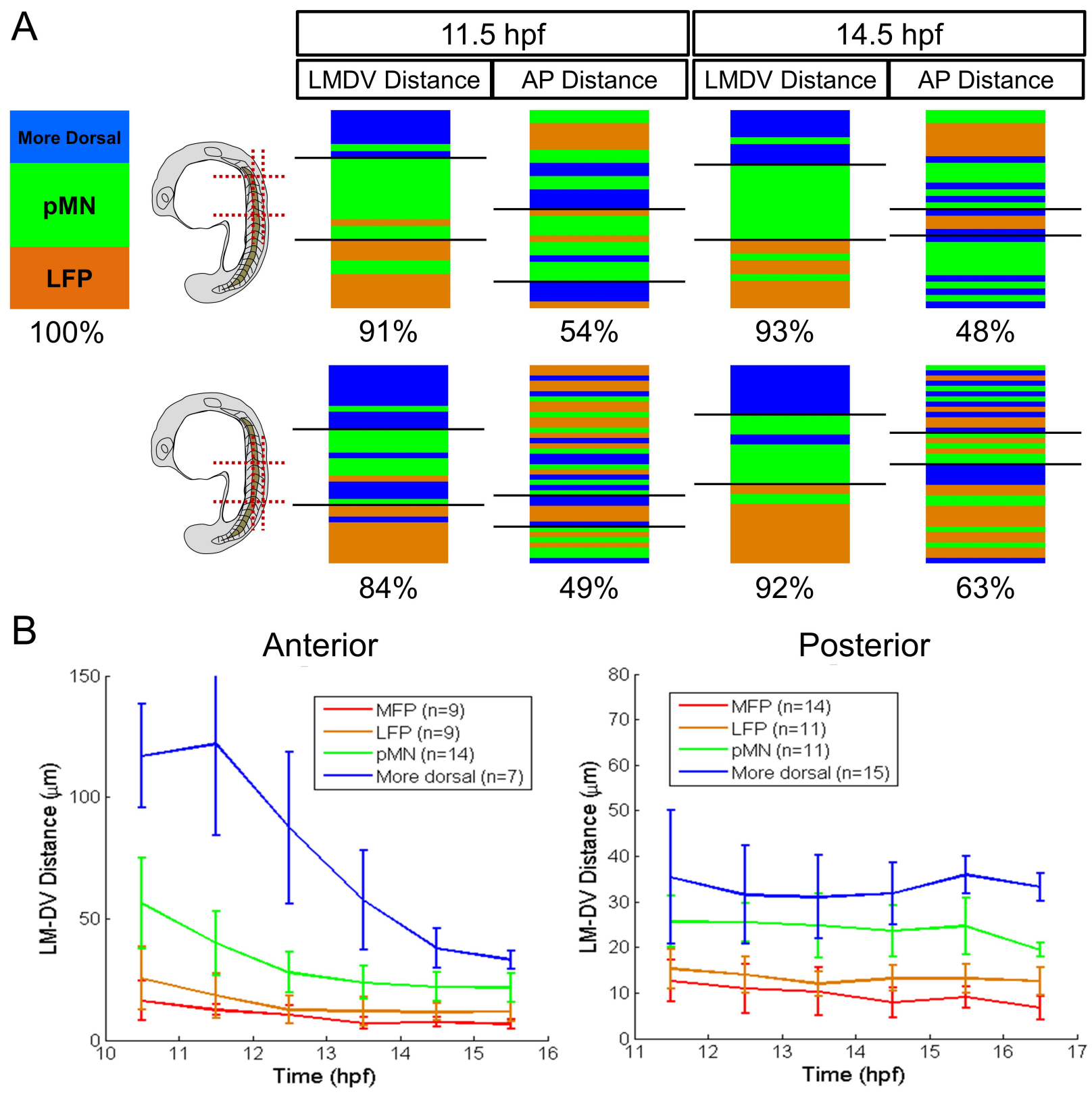

Figure 5. Correlations between position and fate choice in single cells.

(A) Flags drawn using lateral-medial/dorsal-ventral (LMDV) and anterior-posterior (AP) positions as a control for the correlational analysis. Data from an anterior movie and a posterior movie are compared (schematics). The black lines on the flag mark thresholds that best separate different fates. See descriptions in the text and also methods.

(B) Average position tracks. Note the range of anterior progenitors vs. posterior ones over the same time window, indicating different tissue geometry and cell dynamics along the body axis. LMDV: Lateral Medial-Dorsal Ventral distances. Error bars are S.D. 
We drew flags for the same sets of cell tracks by the maximum transient level, total time, and average levels of Shh response (Figure 6A), when ventral neural progenitors are known to become specified (Lewis and Eisen, 2003; Park et al., 2004; Xiong et al., 2013). For the anterior tracks, all metrics are $>85 \%$ correlative with fate choices, consistent with previous population level studies in amniotes (Ericson et al., 1997; Dessaud et al., 2007; Dessaud et al., 2010). Interestingly, for the posterior tracks, only maximum transient level correlates with fate choice for $>80 \%$ of the tracks and the other two metrics fail to make $70 \%$. This difference of correlation percentage is robust to wide variations in values of parameters used in our quantification (e.g. estimated Kaede mRNA half-life, smoothing parameters used in intensity calculation and transcription activity fitting) (Figures 6B-C). Other metrics (except LMDV cell position at late times, which is one of our fate identifiers) do not correlate better than the maximum transient level when both anterior and posterior tracks are considered (Figure 6D, data not shown). No single metric makes perfect predictions. 


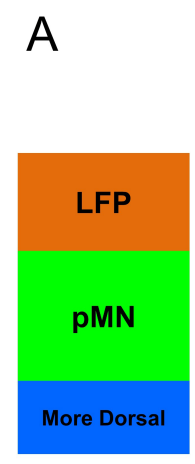

$100 \%$
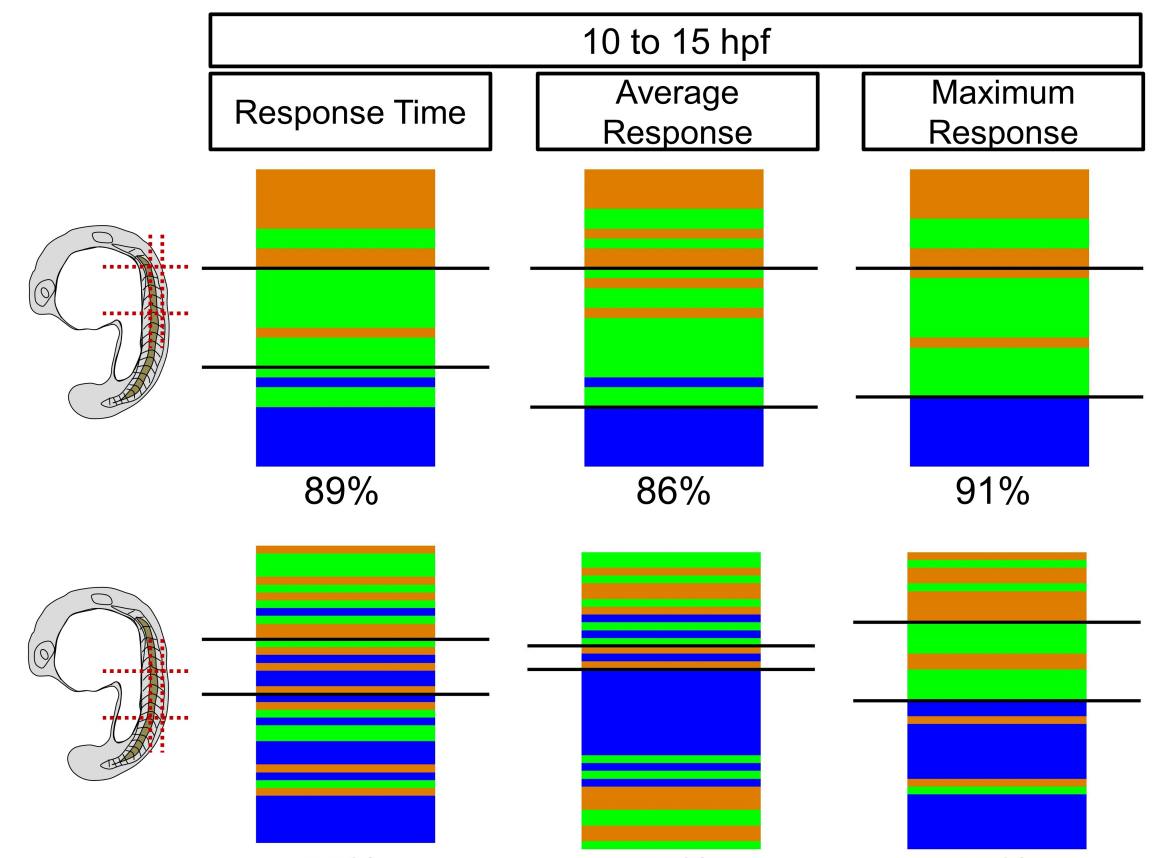

$63 \%$

B

Anterior
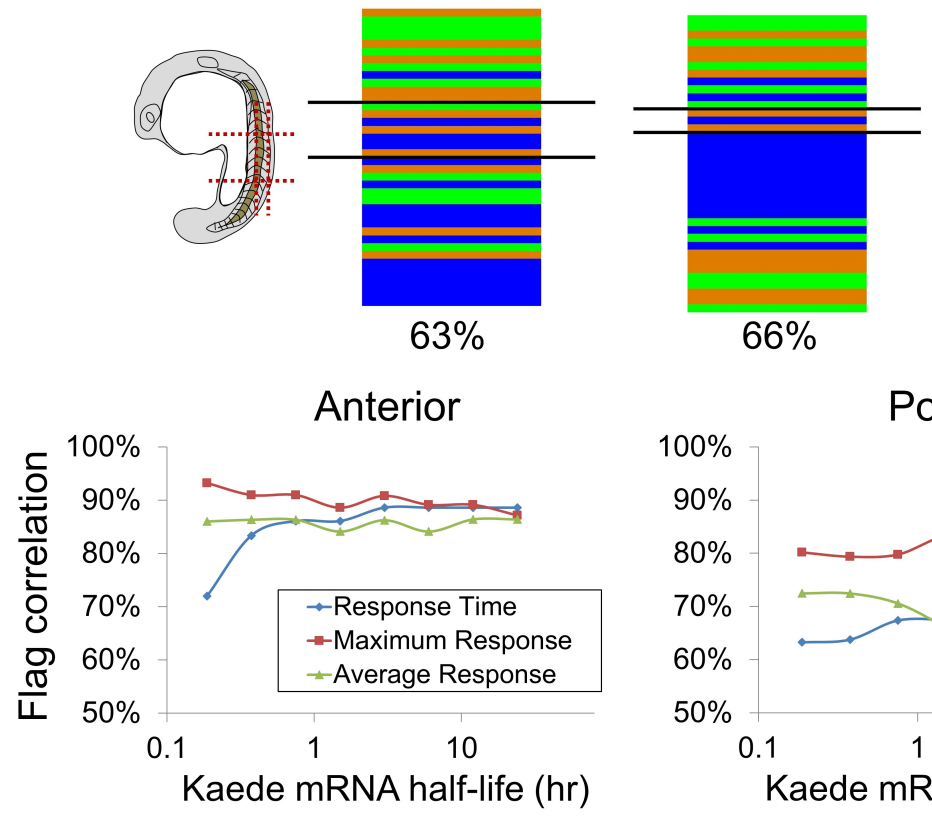

$66 \%$

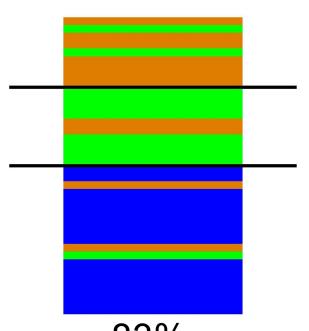

$83 \%$

C
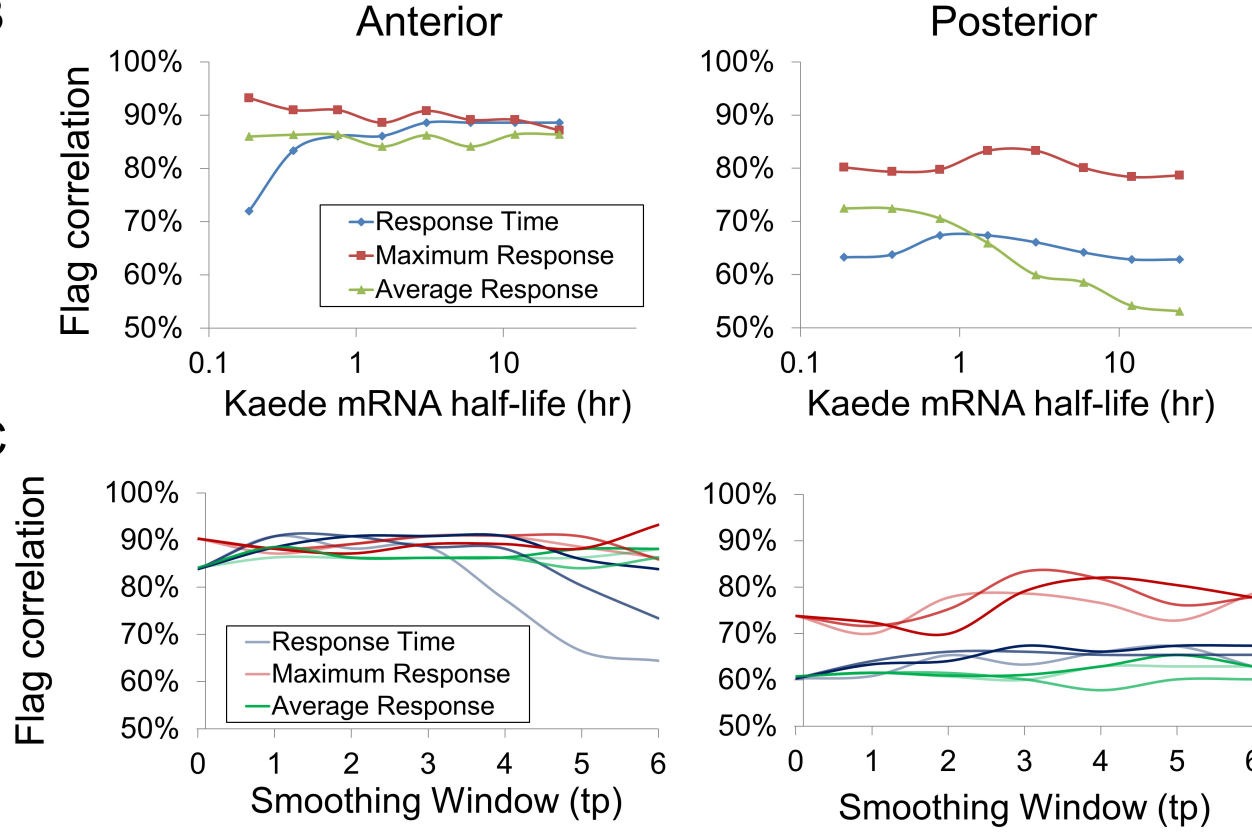

D

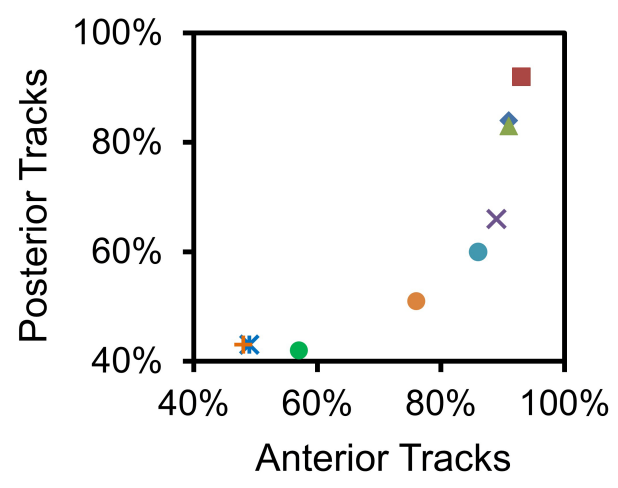

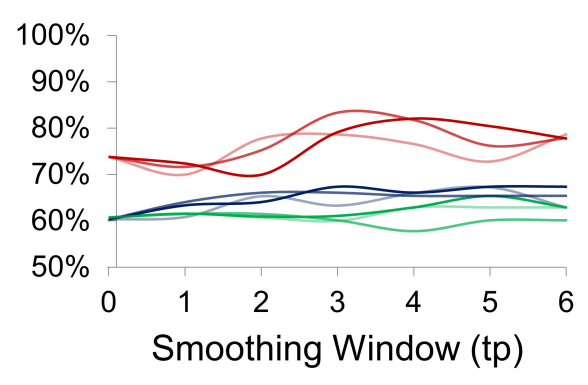

- Early LMDV Position

- Late LMDV Position

$\triangle$ Maximum Response

$\times$ Response Time

- Average Response

- Early Speed of Cells

- Late Speed of Cells

* Early AP Position

+ Late AP Position 
Figure 6. Correlations between different metrics of Shh response and fate choice in single cells.

(A) Shh response dynamics metrics. Under the modeled activity of the ptch2:kaede promoter, Response time is a count of time points at which ptch2:kaede promoter is "on" (>0.05 A.U./hr ${ }^{2}$ ). Average response is the average promoter activity across the whole time window. Maximum response is the highest promoter activity found in the time window.

(B) Flag correlation results of the 3 metrics in (A) (for which half-life $=3$ hours was used) over different Kaede mRNA half-life values. Note that response time and average response parameters switch predictive power as Kaede mRNA becomes stable. Maximum transient response level stays best over wide ranges of mRNA stability. The conclusion in $(A)$ is therefore robust to this estimated parameter (Figure 4).

(C) Flag correlation results of the 3 parameters in (A) (where smoothing window=3 time points and iteration number=2) over different smoothing window ( $\mathrm{x}$ axis) and iteration numbers (dark,1;light,2;lighter,3). The conclusion in $(A)$ is therefore robust to this analysis, despite the sensitivity of equation (3) to the smoothing parameters. (D) Summary of correlation percentages for posterior vs. anterior tracks for different morphogen interpretation models. In addition to metrics mentioned in the main text, cell speeds were also plotted here. The speeds were calculated from the positions of the cells in the track for 2-hour window centered on the early (11.5hpf) and late (14.5hpf) time points.

\section{DISCUSSION}

The imaging, cell tracking, and data analysis reported in this study open a fresh perspective for looking at the important classic problem of morphogen interpretation. While it is reassuring that our data (obtained with a distinct technique) on average corroborate numerous previous studies on Shh mediated ventral neural tube patterning, the increased resolution into single cells and wide temporal coverage from when cells start responding to Shh to cell fate-specification reveal a high degree of previously unrecognized heterogeneity. This single cell variability could be due to other factors that differ between cells and their cross-talk with the Shh gene regulatory network (GRN), such as Notch signaling (Huang et al., 2012), or just simply noise that arises in the GRN (Rosenfeld et al., 2005). These possibilities are difficult to distinguish with the existing data, but they pose a challenge to morphogen patterning in general: how to ensure pattern precision despite this high heterogeneity in signal responses?

One possibility is to code morphogen response in a robust way under the given heterogeneity. Through quantifying the transcriptional dynamics at the Shh reporter, we are able to directly assess the correlation between certain proposed information-coding schemes and fate outcome. In the anterior spinal cord, maximum transient level, response time, and average level are all predictive of fates. In the more posterior spinal cord however, our results show that 
the temporal dimension of Shh response (which is part of the response time and the average level) no longer explains cell fate choices as well as the maximum transient level. The cause of this difference might be that there are intrinsic or environmental differences between neural progenitors in the anterior vs. posterior spinal cord, which affect their morphogen interpretation. Supporting this idea, the tissue geometry, and the range and movement of cells in zebrafish neural tube vary greatly along the body axis (Xiong et al., 2013; this study). We also found that all metrics calculated from posterior tracks are less correlated to fates than those of the anterior ones, suggesting an overall noisier environment. This may be because posterior neural progenitors have a less epithelial character and undergo more cell mixing, or because the spatially small Shh gradient as a result of the posterior neural tube's small size introduces more positional noise in signaling. Overall, at least a level of $\sim 10 \%$ of correlation inaccuracy remains for any tested metric, indicating a limitation of simple response-fate models.

While it is tempting to construct more complex models using our single cell data, certain limitations remain that warrant caution. First, our live imaging is limited by the brightness of reporters and the sensitivity of detection. Dynamics that fall below our imaging capacity, especially the Shh response activities before and in the early hours of our tracks, are not as well resolved. Second, due to the constraint of a live embryo undergoing morphogenesis, certain cells cannot be fully tracked throughout the imaging time window. Third, even though most ventral cells are specified and no longer require Shh by the end of our tracks (17-18hpf, Huang et al., 2012; Xiong et al., 2013), some cells may remain unspecified and their fate reporter could further change beyond our detection. These imperfections of our tracks could give misleading results in overly fine-grained tests (e.g., response level within each 1-hour window, data not shown), where a much smaller number of tracks and time points could dominate and thus bias the analysis.

Nevertheless, the results presented here along with our previous study (Xiong et al., 2013) raise an important consideration for the understanding of morphogen interpretation: given the pervasive heterogeneity of single cells in position, timing, and signaling, how is patterning precision achieved? We speculate that precision at the single cell level may not be necessary as the heterogeneity can average out on the population level, and thus important patterning features such as the size of each progenitor domain can remain robust even under single cell noise. In addition, cellular mechanisms such as cell sorting after specification can refine the final 
pattern. Finally, additional mechanisms such as apoptosis can provide means of correction. Further testing of these ideas would require improved imaging capacities (e.g., light-sheet microscopy) and access to multiple nodes of the GRN with more accurate live reporters (e.g., fluorescent protein knock-ins).

\section{ACKNOWLEDGEMENTS}

We thank D. D'India for fish care, K. Mosaliganti, T. Tsai and members of Matlab community for sharing scripts, A. Green, B. Appel and J. Kuwada for transgenic lines, W. Ma, T. Mitchison, A. Schier, and Megason lab members for discussions. This work is supported by NIH R01 GM107733 to S.G.M and Natural Sciences and Engineering Research Council of Canada (NSERC) to P.H. Author Contributions: F.X., A.R.T. and S.G.M. conceived this study. F.X. and A.R.T. performed the experiments and analyzed the data. T.W.H. performed hsp70l:EGFP experiments and contributed to data analysis. P.H. contributed data of ptch2:kaede line. F.X. and S.G.M. prepared the manuscript with input from all authors. The authors declare no competing financial interest.

\section{REFERENCES}

Ando, R., Hama, H., Yamamoto-Hino, M., Mizuno, H., and Miyawaki, A. (2002). An optical marker based on the UV-induced green-to-red photoconversion of a fluorescent protein. Proc. Natl. Acad. Sci. U.S.A. 99, 12651-12656.

Balaskas, N., Ribeiro, A., Panovska, J., Dessaud, E., Sasai, N., Page, K.M., Briscoe, J., and Ribes, V. (2012). Gene regulatory logic for reading the Sonic Hedgehog signaling gradient in the vertebrate neural tube. Cell 148, 273-284.

Briscoe, J., Pierani, A., Jessell, T.M., and Ericson, J. (2000). A homeodomain protein code specifies progenitor cell identity and neuronal fate in the ventral neural tube. Cell 101, 435-445.

Dessaud, E., Yang, L.L., Hill, K., Cox, B., Ulloa, F., Ribeiro, A., Mynett, A., Novitch, B.G., and Briscoe, J. (2007). Interpretation of the sonic hedgehog morphogen gradient by a temporal adaptation mechanism. Nature 450, 717-720. 
Dessaud, E., Ribes, V., Balaskas, N., Yang, L.L., Pierani, A., Kicheva, A., Novitch, B.G., Briscoe, J., and Sasai, N. (2010). Dynamic assignment and maintenance of positional identity in the ventral neural tube by the morphogen sonic hedgehog. PLoS Biol. 8, e1000382.

Driever, W., and Nüsslein-Volhard, C. (1989). The bicoid protein is a positive regulator of hunchback transcription in the early Drosophila embryo. Nature 337, 138-143.

Driever W, Thoma G, Nüsslein-Volhard C. (1989). Determination of spatial domains of zygotic gene expression in the Drosophila embryo by the affinity of binding sites for the bicoid morphogen. Nature 340, 363-367.

Elowitz, M.B., and Leibler, S. (2000). A synthetic oscillatory network of transcriptional regulators. Nature 403, 335-338.

Ericson, J., Rashbass, P., Schedl, A., Brenner-Morton, S., Kawakami, A., van Heyningen, V., Jessell, T.M., and Briscoe, J. (1997). Pax6 controls progenitor cell identity and neuronal fate in response to graded Shh signaling. Cell 90, 169-180.

Flanagan-Steet, H., Fox, M.A., Meyer, D., and Sanes, J.R. (2005). Neuromuscular synapses can form in vivo by incorporation of initially aneural postsynaptic specializations. Development $132,4471-4481$.

Halloran, M.C., Sato-Maeda, M., Warren, J.T., Su, F., Lele, Z., Krone, P.H., Kuwada, J.Y., and Shoji, W. (2000). Laser-induced gene expression in specific cells of transgenic zebrafish.

Development 127, 1953-1960.

Huang, P., Xiong, F., Megason, S.G., Schier, A.F. (2012). Attenuation of Notch and Hedgehog signaling is required for fate specification in the spinal cord. PLoS Gen 8(6), e1002762.

Jeong, J., and McMahon, A.P. (2005). Growth and pattern of the mammalian neural tube are governed by partially overlapping feedback activities of the hedgehog antagonists patched 1 and Hhip1. Development 132, 143-154.

Jessell, T.M. (2000). Neuronal specification in the spinal cord: inductive signals and transcriptional codes. Nat. Rev. Genet. 1, 20-29.

Kimmel, C., Ballard, W., Kimmel, S., Ullmann, B., and Schilling, T. (1995). Stages of embryonic development of the zebrafish. Dev. Dyn.203, 253-310. 
Lek, M., Dias, J.M., Marklund, U., Uhde, C.W., Kurdija, S., Lei, Q., Sussel, L., Rubenstein, J.L., Matise, M.P., Arnold, H.H., Jessell, T.M., and Ericson, J. (2010). A homeodomain feedback circuit underlies step-function interpretation of a Shh morphogen gradient during ventral neural patterning. Development 137, 4051-4060.

Lewis, K.E., and Eisen, J.S. (2003). From cells to circuits: development of the zebrafish spinal cord. Prog. Neurobiol. 69, 419-449.

Megason, S. (2009). Methods in Molecular Biology. Methods Mol. Biol.546, 317-332.

Ng, A.N., de Jong-Curtain, T.A., Mawdsley, D.J., White, S.J., Shin, J., Appel, B., Dong, P.D., Stainier, D.Y., and Heath, J.K. (2005). Formation of the digestive system in zebrafish: III. Intestinal epithelium morphogenesis. Dev. Biol. 286, 114-135.

Oosterveen, T., Kurdija, S., Alekseenko, Z., Uhde, C.W., Bergsland, M., Sandberg, M., Andersson, E., Dias, J.M.M., Muhr, J., and Ericson, J. (2012). Mechanistic differences in the transcriptional interpretation of local and long-range Shh morphogen signaling. Dev. Cell 23, 1006-1019.

Park, H.C., Shin, J., and Appel, B. (2004). Spatial and temporal regulation of ventral spinal cord precursor specification by Hedgehog signaling. Development 131, 5959-5969.

Rieger, T. R., Morimoto, R. I., \& Hatzimanikatis, V. (2005). Mathematical modeling of the eukaryotic heat-shock response: dynamics of the hsp70 promoter. Biophysical journal 88(3), 1646-1658.

Rosenfeld, N., Young, J.W., Alon, U., Swain, P.S. and Elowitz, M.B., 2005. Gene regulation at the single-cell level. Science, 307(5717), pp.1962-1965.

Shin, J., Park, H.-C., Topczewska, J.M., Mawdsley, D.J., and Appel, B. (2003) Neural cell fate analysis in zebrafish using olig2 BAC transgenics. Meth. Cell Sci. 25(1-2): 7-14.

Shkumatava, A., Fischer, S., Muller, F., Strahle, U., and Neumann, C.J. (2004). Sonic hedgehog, secreted by amacrine cells, acts as a short-range signal to direct differentiation and lamination in the zebrafish retina. Development 131, 3849-3858.

Wolpert, L. (1969). Positional information and the spatial pattern of cellular differentiation. J. Theor. Biol. 25, 1-47. 
Xiong, F., Ma, W., Hiscock, T.W., Mosaliganti, K.R., Tentner, A.R., Brakke, K.A., Rannou, N., Gelas, A., Souhait, L., Swinburne, I.A., et al. (2014). Interplay of cell shape and division orientation promotes robust morphogenesis of developing epithelia. Cell 159, 415-427.

Xiong, F., and Megason, S.G. (2015). Abstracting the principles of development using imaging and modeling. Integr Biol (Camb) 7, 633-642.

Xiong, F., Tentner, A.R., Huang, P., Gelas, A., Mosaliganti, K.R., Souhait, L., Rannou, N., Swinburne, I.A., Obholzer, N.D., Cowgill, P.D., et al. (2013). Specified neural progenitors sort to form sharp domains after noisy Shh signaling. Cell 153, 550-561.

MOVIES

Movie S1. h2b-ebfp2, ptch2:kaede/nkx2.2:mgfp dataset

3D rendered maximum projections. Top view and cross-sectional view. Nuclear EBFP2 label allows easier tracking and identification of $n k x 2.2: m g f p$ cells.

Movie S2. Slice movie showing a single pMN track in a mem-ebfp2, ptch2:kaede/mnx1:gfp dataset

Using a track as input, the movie was automatically generated by re-slicing the dataset centering on the tracked cells (marked by the white dot). The slices thereby allow visual observation of the cell's behavior even as it changes position. This cell is seen to move towards the midline and turn on the GFP reporter, adopting a pMN fate. Top view and cross-sectional view. Time stamp: hh:mm. Scale bar: $10 \mu \mathrm{m}$. Same below.

Movie S3. mem-ebfp2, ptch2:kaede/olig2:gfp anterior dataset

Anterior neural tube formation. Epiboly movement and convergence are apparent in the beginning of the movie.

\section{Movie S4. mem-ebfp2, ptch2:kaede/olig2:gfp posterior dataset}

Posterior neural tube formation. Blastopore closure is apparent in the beginning of the movie.

Tissue is overall smaller than the anterior counterpart (Movies S3). 


\section{MATERIALS AND METHODS}

\section{Zebrafish strains and maintenance}

Tg(shh:gfp) (Shkumatava et al., 2004), tgBAC(ptch2:kaede) (Huang et al., 2012), tg(nkx2.2a:mgfp) (Ng et al., 2005), tg(olig2:gfp) (Shin et al., 2003), tg(mnx1:gfp) (FlanaganSteet et al., 2005) and tg(hsp70l:EGFP) (Halloran et al., 2000) have been described.

Homozygote parents from two different lines were paired to produce double transgenic embryos for imaging. Natural spawning was used and time of fertilization was recorded at the single cell stage of each clutch. Embryos were kept in $28^{\circ} \mathrm{C}$ incubators/chambers during imaging and other times except room temperature during injections, dechorionating and mounting. Staging was recorded using morphological criteria and aligned to the normal table (Kimmel et al, 1995). All fish are housed in fully equipped and regularly maintained and inspected aquarium facilities. Fish-related protocols have been approved by the Institutional Animal Care and Use Committee (IACUC) at Harvard Medical School.

Microinjections of mRNAs

A pMTB construct (Xiong et al., 2013) containing mem-EBFP2 or h2b-EBFP2 was used for mRNA synthesis with the mMESSAGE mMACHINE system (Ambion). For mosaic injections, one blastomere of 8 to 16-cell stage embryos was injected (Nanoject) with approximately $1 \mathrm{nl}$ of 20ng/ $\mu \mathrm{l}$ mem-EBFP2 or h2b-EBFP2. One round of screening was applied immediately after mosaic injections to eliminate damaged embryos and/or ones that missed the injection (no retention of co-injected Phenol Red label). Before imaging, injected embryos were screened for health.

\section{Timelapse confocal imaging}

Live imaging was performed using a Zeiss 710 confocal microscope (objective: C-Apochromat 40X $1.2 \mathrm{NA}$ ) with a home-made heating chamber maintaining $28^{\circ} \mathrm{C}$. Embryos were mounted using the dorsal mount (Megason 2009) with a stereoscope (Leica MZ12.5). The mounting steps for imaging the neural plate/tube have been described in detail in Xiong et al., 2013. $405 \mathrm{~nm}$ laser was used for photo conversion of Kaede and excitation of EBFP2 at the same time. $488 \mathrm{~nm}$ and $561 \mathrm{~nm}$ were then used in a separate track for the GFP and Kaede(Red) signals (See also Figure 1B). A typical stack covers an imaging space of $303 \mu \mathrm{m}(\mathrm{x}), 303 \mu \mathrm{m}(\mathrm{y})$ and 
$120 \mu \mathrm{m}(\mathrm{z})$, divided by $700 \times 700 \times 80$ voxels and is taken within 6 minutes. A typical movie lasts 10 hours containing $\sim 100$ stacks at 6 minute intervals.

Data analysis

For the present study 31 timelapse datasets were collected (29 contain ptch2:kaede with olig2:gfp:15; nkx2.2:mgfp:6; shh:gfp:4; mnx1:gfp:4. 2 contain olig2:dsRed and nkx2.2:mgfp). 21/31 allow cell tracking and 13/21 have high coverage. Tracks were generated for 7/13 and 4/7 were further processed. The datasets acquired in Zeiss .Ism format were first converted to Megacapture format for import into GoFigure2 software (www.gofigure2.org, see also Xiong et al., 2013; Xiong et al., 2014). Inside GoFigure2, segmentation and tracking were performed manually on each dataset on randomly selected ventral neural tube cells in the labeled embryo (e.g., Figures 1D-E). To calculate the cell's positions in relation to the embryo, notochord segmentations were generated every 10 time points (1 hour in real time) for each presented dataset. Segmentation and track tables exported from GoFigure2 were further processed using custom scripts (provided in accompanying supplemental data files) to generate the single slice labeled movies (Movie S2). The movies were watched individually to determine the fate choice of the cell using a combinatorial criteria including fate reporter expression and final position in the ventral neural tube, as described in Xiong et al., 2013. First, at 16hpf, MFPs were identified as the centered, apically constricted, triangle-shaped column of cells immediately dorsal to the notochord (as illustrated in Figure 1A). This fate identification is checked with shh:gfp movies in which MFPs are GFP+. Next, LFPs are identified as the two columns of cells flanking both sides of the MFP (Huang et al., 2012). This fate identification is confirmed with nkx2.2:mgfp movies (e.g., Movie S1). Next, pMNs are identified as the cells dorsal to the floor plate cells that are strongly olig2:gfp positive. Finally, More dorsal cells are those that are further dorsal to the pMN domain and GFP- in olig2:gfp movies or that are located $>40 \%$ ventral dorsal distance at 16hpf in movies without olig2:gfp signal. The segmentations, tracks and cell fate information were then assembled through a preprocessing pipeline (provided in accompanying supplemental data files) in Matlab (Mathworks). The output of the pipeline is a set of Matlab matrixes containing organized raw data for downstream analysis (e.g., NewFinalWrkSpace olig2 9.mat, see supplemental data files). This organized raw data was then processed through a series of filters (provided in accompanying supplemental data files) to account for multiple experimental factors that introduce small errors in intensity measurements including bleed-through, saturation, and 
noise (e.g. SaturationMask.m: a first filter that removes aberrations in intensity calculation arising from saturated pixels in the image. BleedthroughCorr.m: correcting bleed-through signal between two channels. Bleed-through correction runs automatically after the algorithm assesses the degree of bleed-through and then applies a percentage subtraction on the values to further reduce errors in intensity calculation. SmoothData.m: a smoothing filter that handles short-time fluctuations that come from segmentation variations and acquisition noise. Different utility scripts were coded to allow user interactions with both the raw and filtered data, listed in AnalysisScript List.xlsx in accompanying supplemental data files. Full analysis protocols, scripts and sample datasets are collectively provided as a package of supplemental data files available for download here:

https://wiki.med.harvard.edu/pub/SysBio/Megason/Publications/NT2 Supplemental DataFiles.zi 